\title{
Bayesian Estimation of Medium Properties in Wavefield Downward Extrapolation Problems
}

\author{
IOANNIS PITAS, MEMBER, IEEE, AND ANASTASIOS N. VENETSANOPOULOS, SENIOR MEMBER, IEEE
}

\begin{abstract}
Acoustic waves are used in many areas (ultrasonic medicine, geophysical oil prospecting, etc.) for nondestructive imaging of the interior of objects such as the earth, the human body, etc. The wavefield measurements recorded on the surface of the object are extrapolated according to the wave equation to give an "image" of the object by using an imaging principle. The extrapolation propagates backward the noise present in the measurements. Thus, the quality of the final image is deteriorated, unless statistical restoration techniques are used. Another source of degradation of the image is the insufficient knowledge of some properties of the medium such as the wave propagation velocity. The aim of this work is to use estimation theory to determine good estimates of the velocity and of the backward extrapolated wavefield from the known surface wavefield measurements.
\end{abstract}

\section{INTRODUCTION}

$\mathrm{W}^{\mathrm{s}}$ AVEFIELD measurements and the wave equation are used in many disciplines such as geophysical exploration [1]-[3], ultrasonic imaging in medicine [5], and physical and geometric optics [6]. Many of the applications in these areas are essentially nondestructive imaging of the interior of certain objects by means of the wave energy. Waves are produced by sources on the surface of the object and transmitted through the object. The reflected wavefield is measured and recorded on the surface of the object. One way to obtain an image of the interior of the object is to extrapolate the data backwards and to use an imaging principle afterwards. This technique consists of two steps: 1) wavefield backward extrapolation, and 2) imaging. Step 1) uses the wave equation to find the wavefield at depth $z_{0}$ in the interior of the earth. Step 2) collects the results of the first step around zero travel time $(t=0)$ to find the distribution of the reflector points at depth $z_{0}$ [1]. This extrapolation-imaging technique has been used successfully in seismic oil prospecting [1]-[3] and in ultrasonic imaging [5]. In the following, we shall concentrate on the wavefield backward extrapolation because it is the most complicated and difficult step and its results are usually deteriorated either by the presence of noise or by the imprecise knowledge of the wave propagation velocity.

Manuscript received August 8, 1986; revised November 6, 1987. This work was supported by the Natural Sciences and Engineering Research Council of Canada under Grant 1236.

I. Pitas is with the Department of Electrical Engineering, University of Thessaloniki, Thessaloniki 540 06, Greece, on leave at the Department of Electrical Engineering, University of Toronto, Toronto, Ont,, Canada.

A. N. Venetsanopoulos is with the Department of Electrical Engineering, University of Toronto, Toronto, Ont., Canada.

IEEE Log Number 8719422
The recorded data are usually corrupted by noise. nis noise can be white additive Gaussian noise (such as background noise) or colored Gaussian noise (such as surtice waves carrying no information about the interior of the object). The white noise is usually reduced during the processing of the wavefield measurements. In seismic oil prospecting, this noise is removed by stacking [2], [3] different seismic traces or by a simple low-pass filtering. The colored noise is usually suppressed by velocity filtering [7], since the surface waves usually have smaller velocity than the reflected or scattered ones. These techniques usually distort the data. The surface wavefield data are then extrapolated backwards and give the image of the object by using an imaging principle. The remaining coherent noise usually has a bad effect on the quality of the image.

Another source of degradation of the image is the imprecise knowledge of the wave propagation velocity. The velocity is usually estimated from the surface measurements [3] or it is measured by other experiments (e.g., by VSP methods). However, both methods give inaccuracies in the velocity measurement. These inaccurate velocities are used in the extrapolation process and deteriorate the quality of its results. Thus, more sophisticated techniques have to be used for better noise suppression and better wavefield extrapolation. The Wiener filtering is such a technique [9] which has been applied in seismic migration [10], in potential data extrapolation [2], and in ultrasonic imaging [11]. Wiener filtering gives better results than simple noise filtering followed by wavefield extrapolation. However, it cannot take into account velocity inaccuracies. Our approach is to make-a joint estimation of the extrapolated field and of the wave velocity based on the surface measurements. Such an approach couples wave theory and estimation theory [12]. Estimation theory has already been used in inversion problems [13], [14]. The wavefield inversion examples given in [13] treat the problem of earthquake hypocenter location. Their analysis is based on the ray path method. The novelty of our analysis is that it couples wave theory with statistical estimation and that it makes simultaneous estimation of the extrapolated field and of the wave velocity. Our approach can be easily extended to the multilayered media [20] for seismic migration purposes.

The contents of this paper are now summarized. A brief outline of the basics of the wave equation and the problem 
statement are given in Section II. The maximum likelihood (ML) estimation method is analyzed in Section III. Maximum a posteriori (MAP) estimation is analyzed in Section IV. Examples follow in Section V and conclusions are drawn in Section VI.

\section{Problem Statement}

The acoustic wave equation

$$
\nabla^{2} p-\frac{1}{c^{2}} \frac{\partial^{2} p}{\partial t^{2}}=0
$$

has the following solution for upward traveling plane waves [2], [3] in a homogeneous medium in the wavenumber-frequency domain:

$$
P\left(k_{x}, k_{y}, z=0, \omega\right)=P\left(k_{x}, k_{y}, z_{0}, w\right) H\left(k_{x}, k_{y}, z_{0}, \omega\right)
$$

where

$$
\begin{aligned}
H\left(k_{x}, k_{y}, z_{0}, \omega\right) & =\left\{\begin{array}{c}
\exp \left(-i z_{0} \sqrt{k^{2}-k_{x}^{2}-k_{y}^{2}}\right) \\
k^{2} \geq k_{x}^{2}+k_{y}^{2} \\
\exp \left(-z_{0} \sqrt{k_{x}^{2}+k_{y}^{2}-k^{2}}\right) \\
k^{2}<k_{x}^{2}+k_{y}^{2}
\end{array}\right. \\
k & =\omega / c .
\end{aligned}
$$

Thus, the wavefield $P\left(k_{x}, k_{y}, z=0, \omega\right)$ (Fig. 1) on the object surface $(z=0)$ can be calculated by upward extrapolation of the wave $P\left(k_{x}, k_{y}, z=z_{0}, \omega\right)$ on the surface $z=z_{0}$ through the operator $H\left(k_{x}, k_{y}, z_{0}, \omega\right)$. The wave propagation velocity is denoted by $c$. Equation (2) has the following formula in the space-frequency domain:

$$
\begin{aligned}
P(x, y, z=0, \omega)= & P\left(x, y, z=z_{0}, \omega\right) \\
& * H\left(x, y, z_{0}, \omega\right)
\end{aligned}
$$

where $*$ denotes the two-dimensional convolution and

$$
\begin{aligned}
H\left(x, y, z_{0}, \omega\right) & =\frac{z_{0}}{2 \pi} \frac{1+i k r}{r^{3}} e^{-i k r} \\
r & =\sqrt{x^{2}+y^{2}+z_{0}^{2}} .
\end{aligned}
$$

Equations (4) and (5) constitute the Rayleigh integral [2].

The upward wave extrapolation in the space-time domain has the following form:

$$
\begin{aligned}
p(x, y, z=0, t) & =p\left(x, y, z=z_{0}, t\right) * h\left(x, y, z_{0}, t\right) \\
h\left(x, y, z_{0}, t\right) & =\frac{\partial}{\partial z} \frac{\delta(t-r / c)}{-2 \pi r} .
\end{aligned}
$$

$\delta$ is a delta function and $*$ denotes a three-dimensional convolution. Formulations (2), (4), (6) are fully equivalent.

If the surface acoustic field is known, the acoustic field at depth $z=z_{0}$ can be found by downward extrapolation:

$$
\begin{aligned}
& P\left(k_{x}, k_{y}, z=z_{0}, \omega\right) \\
& \quad=P\left(k_{x}, k_{y}, z=0, \omega\right) W\left(k_{x}, k_{y}, z_{0}, \omega\right) .
\end{aligned}
$$

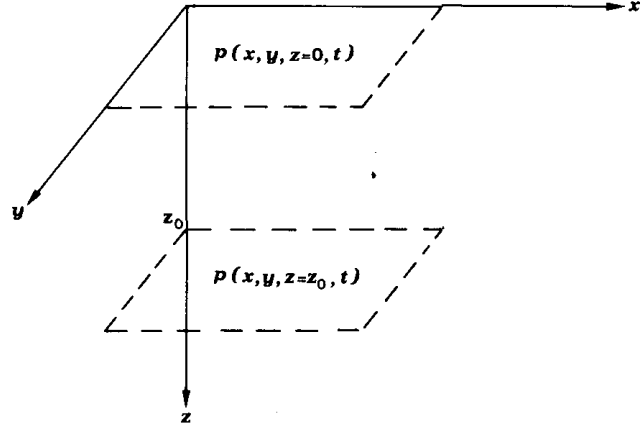

Fig. 1. Upward propagation of plane acoustic waves in a homogeneous medium.

The downward extrapolation operator $W\left(k_{x}, k_{y}, z_{0}, \omega\right)$ is the inverse of the upward extrapolation operator $H\left(k_{x}, k_{y}\right.$, $\left.z_{0}, \omega\right)$ :

$$
W\left(k_{x}, k_{y}, z_{0}, \omega\right)=\left\{\begin{array}{c}
\exp \left(i z_{0} \sqrt{k^{2}-k_{x}^{2}-k_{y}^{2}}\right) \\
k^{2} \geq k_{x}^{2}+k_{y}^{2} \\
\exp \left(z_{0} \sqrt{k_{x}^{2}+k_{y}^{2}-k^{2}}\right) \\
k^{2}<k_{x}^{2}+k_{y}^{2} .
\end{array}\right.
$$

Operator $W\left(k_{x}, k_{y}, z_{0}, \omega\right)$ is unstable because of the lower part of (8), which is of high-pass nature. Therefore, the following matched inversion operator [1] is used in practice for downward extrapolation:

$$
W^{\prime}\left(k_{x}, k_{y}, z_{0}, \omega\right)=\left\{\begin{array}{c}
\exp \left(i z_{0} \sqrt{k^{2}-k_{x}^{2}-k_{y}^{2}}\right) \\
k^{2} \geq k_{x}^{2}+k_{y}^{2} \\
\exp \left(-z_{0} \sqrt{k_{x}^{2}+k_{y}^{2}-k^{2}}\right) \\
k^{2}<k_{x}^{2}+k_{y}^{2}
\end{array}\right.
$$

The knowledge of the correct wave propagation velocity is needed for the application of the operator (9). If the wrong propagation velocity is used, the results of the downward wavefield extrapolation are not well focused [8]. Thus, the determination of the correct wave propagation velocity is crucial for the quality of the results of the downward wavefield extrapolation.

If observation noise is present in the surface wavefield data $p(x, y, z=0, t)$, the following equation describes the upward wave expolation:

$$
\begin{aligned}
& p(x, y, z=0, t) \\
& \quad=p\left(x, y, z=z_{0}, t\right) * h\left(x, y, z_{0}, t\right)+n(x, y, t) .
\end{aligned}
$$

The observation noise $n(x, y, t)$ may be white noise or surface plane waves coming from the seismic source. Two techniques are known for the removal of the observation noise. The first one is the velocity filtering [7], which is used for the removal of direct surface waves. This technique removes the noise, but sometimes distorts the data. 
The second technique is Wiener filtering [10], which uses the following operator for the downward extrapolation:

$$
\begin{array}{r}
W_{\text {Wiener }}\left(k_{x}, k_{y}, z_{0}, \omega\right) \\
=\frac{H^{*}\left(k_{x}, k_{y}, z_{0}, \omega\right)}{\left|H\left(k_{x}, k_{y}, z_{0}, \omega\right)\right|^{2}+\left|\frac{S_{n n}\left(k_{x}, k_{y}, \omega\right)}{S_{p p}\left(k_{x}, k_{y}, \omega\right)}\right|^{2}}
\end{array}
$$

where $S_{n n}\left(k_{x}, k_{y}, \omega\right)$ and $S_{p p}\left(k_{x}, k_{y}, \omega\right)$ denote the power spectra of the observation noise $n(x, y, t)$ and of the wavefield $p\left(x, y, z=z_{0}, t\right)$, respectively. Wiener filtering is efficient in removing observation noise effects in downward extrapolation. Its only drawback is that it requires the knowledge of the correct propagation velocity $c$.

Now we can formulate the problem of the noisy acoustic wavefield extrapolation in the following way.

Given the noisy acoustic surface wavefield measurements $p(x, y, z=0, t)$, estimate the wavefield $p(x, y, z$ $\left.=z_{0}, t\right)$ at depth $z=z_{0}$ and the wave propagation velocity c.

The problem as it stands is ill posed and it has no unique solution. However, additional criteria (e.g., maximization of the a posteriori probability) can reduce the ambiguity of the solution. Such an approach will be followed in the solution of the problem. For simplicity of notation, we shall use the following matrix notation for the discrete version of (10):

$$
y=H x+n .
$$

The boldface letters $\boldsymbol{y}, \boldsymbol{x}, \boldsymbol{n}$ denote vectors which have as elements the values of $p(i \Delta x, j \Delta y, z=0, k \Delta t), p(i \Delta x$, $\left.j \Delta y, z=z_{0}, k \Delta t\right), n(i \Delta x, j \Delta y, k \Delta t)$, respectively, in a lexicographic order. Vectors $\boldsymbol{x}, \boldsymbol{y}$ must not be confused with the spatial coordinates $x$ and $y . \Delta x, \Delta y, \Delta t$ are the sampling intervals in the axes $x, y, t$, respectively. Without loss of generality, we assume that $0 \leq i \leq N_{x}-1$, $0 \leq j \leq N_{y}-1,0 \leq k \leq N_{t}-1$. Therefore, vectors $\boldsymbol{x}$, $\boldsymbol{y}, \boldsymbol{n}$ have dimension $N=N_{x} N_{y} N_{t}$. The matrix $H$ stands for the convolution operator $h\left(i \Delta x, j \Delta y, z_{0}, k \Delta t\right)$. Its dimension is $N \times N$. The space-time formulation of the problem in (12) is convenient only for derivation of the problem solutions. In practice, every convolution operation will be performed in the wavenumber-frequency domain, as will be seen in the examples.

Having defined the problem, we proceed to the maximum likelihood estimation.

\section{Maximum Likelihood Estimation}

Maximum likelihood estimation is a well-known technique [12] applied in scientific areas such as communications [12], inverse problems [13], [14], and seismic deconvolution [15]. It makes use of the "likelihood function" $l(\boldsymbol{x}, c \mid \boldsymbol{y})$ of the unknown $\boldsymbol{x}, c$ given the noisy measurements $y$. This function is proportional to the probability density function (pdf) of the measurements given the parameters $x, c$ :

$$
l(\boldsymbol{x}, c \mid y) \propto p(\boldsymbol{y} \mid \boldsymbol{x}, c) .
$$

When the pdf $p(y \mid x, c)$ is Gaussian, it is better to work with the natural logarithm of $l(x, c \mid y)$ than with $l(x$, $c \mid \boldsymbol{y})$ itself:

$$
L(x, c \mid y) \triangleq \ln l(x, c \mid y) .
$$

The ML estimates $\hat{\boldsymbol{x}}, \hat{c}$ of $\boldsymbol{x}, c$ are the ones which maximize the log-likelihood function (14). In our case, the pdf $p(\boldsymbol{y} \mid \boldsymbol{x}, c)$ is the same as the pdf of the noise $p(\boldsymbol{n})$, as can be easily seen from (12). We shall assume that the noise $\boldsymbol{n}$ is Gaussian with zero mean and covariance matrix $R_{n}$ :

$$
P(n)=\frac{1}{(2 \pi)^{N / 2} R_{n}^{1 / 2}} \exp \left(-\frac{1}{2} n^{T} R_{n}^{-1} n\right) .
$$

By taking the logarithm of (15) and dropping some constant terms, the log-likelihood function becomes

$$
L(x, c \mid y)=-\frac{1}{2}(y-H x)^{T} R_{n}^{-1}(y-H x) .
$$

This function has to be maximized with respect to $x, c$. We set the derivatives of $L(\boldsymbol{x}, c \mid \boldsymbol{y})$ with respect to $\boldsymbol{x}, c$ equal to zero:

$$
\begin{aligned}
\nabla_{x} L & =H^{T} R_{n}^{-1}(y-H x)=0 \\
\frac{\partial L}{\partial c} & =\left(\frac{\partial H}{\partial c} x\right)^{T} R_{n}^{-1}(y-H x)=0
\end{aligned}
$$

where $\partial H / \partial c$ is a matrix having as elements the partial derivatives of the elements of $H$. Equations (17) and (18) give the ML estimators of $\boldsymbol{x}, c$. These equations are both valid if

$$
\boldsymbol{x}=H^{-1} \boldsymbol{y} .
$$

The result is trivial [17] (inverse filter), and besides, it does not suggest a way to estimate $c$. Therefore, we proceed to the maximum a posteriori estimation.

\section{Maximum A Posteriori (MAP) Estimation}

Maximum likelihood estimation does not take into account any a priori information available about the object, its properties, and about the wavefield $x$. Sometimes such information is available in a probabilistic form [16]. Usually the $a$ priori probability density functions $p(c)$ and $p(x)$ are known. We shall assume that velocity $c$ and the field $x$ are statistically independent and that they are normally distributed. Furthermore, the a priori velocity mean $\bar{c}$, the variance $\sigma_{c}^{2}$, the mean wavefield $\bar{x}$, and the autocorrelation matrix $R_{x}$ are supposed to be known. Thus,

$$
\begin{aligned}
p(x)= & \frac{1}{(2 \pi)^{N / 2}\left|R_{x}\right|^{1 / 2}} \\
& \cdot \exp \left\{-\frac{1}{2}(x-\bar{x})^{T} R_{x}^{-1}(x-\bar{x})\right\}
\end{aligned}
$$




$$
p(c)=\frac{1}{\sqrt{2 \pi} \sigma_{c}} \exp \left\{-\frac{(c-\bar{c})^{2}}{2 \sigma_{c}^{2}}\right\} .
$$

All this available information is taken into account in the MAP estimation.

Maximum a posteriori (MAP) estimation optimizes the logarithm of the a posteriori probability density function $p(\boldsymbol{x}, c \mid \boldsymbol{y})$. It is very well known from probability theory that

$$
p(\boldsymbol{x}, c \mid \boldsymbol{y})=\frac{p(\boldsymbol{y} \mid \boldsymbol{x}, c) p(\boldsymbol{x}, c)}{p(\boldsymbol{y})}
$$

where $p(x, c)$ is the joint pdf of $\boldsymbol{x}, c$ and $p(\boldsymbol{y} \mid \boldsymbol{x}, c)$ is the $a$ priori pdf already used in ML estimation. The random variables $\boldsymbol{x}, c$ are statistically independent; therefore,

$$
P(x, c \mid y)=\frac{P(y \mid x, c) P(x) P(c)}{P(y)} .
$$

The function to be optimized with respect to $x, c$ is the following:

$$
\begin{aligned}
L(x, c)= & \ln p(x, c \mid y)=\ln p(y \mid x, c) \\
& +\ln p(x)+\ln p(c)-\ln p(y) .
\end{aligned}
$$

By taking (16), (20), (21), and (23) into account and neglecting constant terms, we have

$$
\begin{aligned}
L(x, c)= & -\frac{1}{2}(y-H x)^{T} R_{n}^{-1}(y-H x) \\
& -\frac{1}{2}(x-\bar{x})^{T} R_{x}^{-1}(x-\bar{x})-\frac{1}{2} \frac{(c-\bar{c})^{2}}{\sigma_{c}^{2}} .
\end{aligned}
$$

Taking the derivatives of $L(x, c)$ equal to zero, we find

$$
\begin{aligned}
\nabla_{x} L & =H^{T} R_{n}^{-1}(y-H x)-R_{x}^{-1}(x-\bar{x})=0 \\
\frac{\partial L}{\partial c}=\left(\frac{\partial H}{\partial c} x\right)^{T} R_{n}^{-1}(y-H x)-\frac{c-\bar{c}}{\sigma_{c}^{2}} & =0 .
\end{aligned}
$$

Thus, the MAP estimators satisfy the equations

$$
\begin{aligned}
& \hat{\boldsymbol{x}}=\overline{\boldsymbol{x}}+R_{x} H^{T} R_{n}^{-1}(y-H \hat{x}) \\
& \hat{c}=\bar{c}+\sigma_{c}^{2}\left(\frac{\partial H}{\partial \hat{c}} \hat{\boldsymbol{x}}\right)^{T} R_{n}^{-1}(y-H \hat{x}) .
\end{aligned}
$$

Equations (28) and (29) cannot be solved explicitly for $\hat{x}$, $\hat{c}$. However, they have a recursive form, and thus can be the basis of a recursive MAP estimator (Picard's iteration). When $c$ is known, then (29) reduces to the wellknown Wiener filter [12], [17] with the a priori mean $\bar{x}$ included:

$$
\begin{aligned}
\hat{\boldsymbol{x}}= & \left(R_{x}^{-1}+H^{T} R_{n}^{-1} H\right)^{-1} R_{x}^{-1} \bar{x} \\
& +\left(R_{x}^{-1}+H^{T} R_{n}^{-1} H\right)^{-1} H^{T} R_{n}^{-1} y .
\end{aligned}
$$

Picard's iteration is not always very stable. The initial estimates $\hat{\boldsymbol{x}}_{0}, \hat{c}_{0}$ also have to be close to the solution $\hat{\boldsymbol{x}}$, $\hat{c}$. Thus, other iteration methods have to be used to minimize $L$ [17]. Such a method is the steepest descent [18]. The basic iteration scheme is the following.

1) Calculate $\nabla_{x} L_{k}$ and $\partial L / \partial c_{k}$ from (26), (27).

2) If $\left\|\nabla_{x} L_{k}\right\|<\epsilon$ and $\left|\partial L / \partial c_{k}\right|<\epsilon$, stop. ( $\epsilon$ is a small positive threshold.)

3) Choose $a_{k}$ to minimize $L\left(x_{k}, c_{k}-a_{k} \partial L / \partial c_{k}\right)$.

4) $c_{k+1}=c_{k}-a_{k}\left(\partial L / \partial c_{k}\right)$.

5) Choose $b_{k}$ to minimize $L\left(x_{k}-b_{k} \nabla_{x} L_{k}, c_{k}\right)$.

6) $x_{k+1}=x_{k}-b_{k} \nabla_{x} L_{k}$.

The variables $x, c$ can be updated simultaneously or one can be updated first (usually $c$ ) until it converges to a solution, and then the other can be updated.

Another possible iterative method can be obtained by substituting (30) in (25). In this case, the function $L(\boldsymbol{x}$, c) becomes

$$
\begin{aligned}
L(c)= & -\frac{1}{2}\left[y-H G^{-1}\left(R_{x}^{-1} \bar{x}+K y\right)\right]^{T} \\
& \cdot R_{n}^{-1}\left[y-H G^{-1}\left(R_{x}^{-1} \bar{x}+K y\right)\right] \\
& -\frac{1}{2}\left[G^{-1}\left(R_{x}^{-1} \bar{x}+K y\right)-\bar{x}\right]^{T} \\
& \cdot R_{x}^{-1}\left[G^{-1}\left(R_{x}^{-1} \bar{x}+K y\right)-\bar{x}\right]-\frac{1}{2 \sigma_{c}^{2}}(c-\bar{c})^{2} \\
G= & R_{x}^{-1}+H^{T} R_{n}^{-1} H \quad K=H^{T} R_{n}^{-1} .
\end{aligned}
$$

The function $L(c)$ has to be optimized with respect to $c$ only. This can be done either by a direct search [18] or by using the gradient $d L / d c$ [18]. When the optimal $c$ is found, the optimal $\boldsymbol{x}$ is calculated from (30).

The solutions given by all the above-mentioned recursive techniques are not unique nor necessarily globally optimal. This comes from the fact that optimization techniques converge sometimes to local minima, rather than to the global minimum of (25). However, the application of the MAP estimation can give good results, as is shown in the examples of Section VI.

\section{Simulation Examples}

The methods described in the previous sections will be illustrated by an example which is commonly used to show the validity of a method (e.g., in [8]). The physical experiment of the example is illustrated in Fig. 2. A diffractor point is located at depth $z_{0}=150 \mathrm{~m}$ in the earth. When a subsurface explosion is made, a wave propagates downward in the earth and it is diffracted by the diffractor point. The diffracted wave is recorded by geophones placed on the surface of the earth. The resulting data record is shown in Fig. 3. The time axis is divided by 2 , so that we have one-way travel times. The measurements are usually corrupted by white background noise such as in Fig. 5, or by surface waves traveling from the explosion to the geophones shown in Fig. 10, or by both of them. The equation describing the experiment is the two-dimensional version of (10) [1]: 


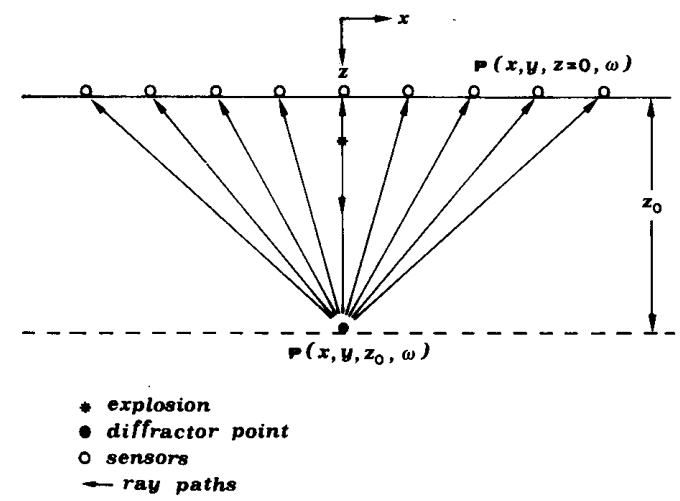

Fig. 2. Subsurface explosion and acoustic wave diffraction.

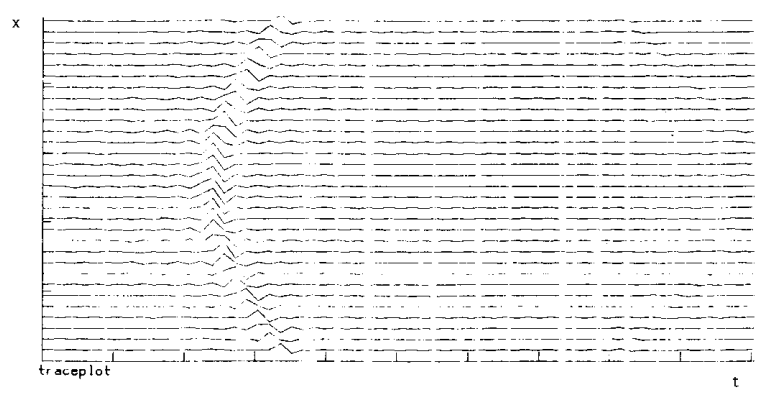

Fig. 3. Seismic traces recorded by geophones in the experiment of Fig. 2.

$$
\begin{gathered}
P(x, z=0, \omega)=P\left(x, z=z_{0}, \omega\right) \\
* H(x, \omega)+N(x, \omega) \\
H(x, \omega)=-\frac{i k}{2} \frac{z}{r} H_{1}^{(2)}(k r) \\
r=\sqrt{x^{2}+z_{0}^{2}} .
\end{gathered}
$$

The operator $H(x, \omega)$ has the following form in the wavenumber-frequency domain:

$$
H\left(k_{x}, k\right)= \begin{cases}\exp \left(-i z_{0} \sqrt{k^{2}-k_{x}^{2}}\right) & k^{2} \geq k_{x}^{2} \\ \exp \left(-z_{0} \sqrt{k_{x}^{2}-k^{2}}\right) & k^{2}<k_{x}^{2} .\end{cases}
$$

The first part of (36) corresponds to the upward propagating waves and the second part to the evanescent waves. For depths $z_{0}$ large enough, the evanescent part is approximately equal to zero.

We shall consider first the case of the white Gaussian noise shown in Fig. 5. The wavefield $p\left(x, z_{0}, t\right)$ to be estimated is shown in Fig. 4. It consists of a perfect impulse at $t=0$, of strength 5 . However, it is assumed to be unknown to the experimenter. Our aim is to estimate the wavefield $p\left(x, z_{0}, t\right)$, denoted by $x$, based on the noisy surface measurements $p(x, z=0, t)$, denoted by $y$, which are given by (34) and are shown in Fig. 5. The noise is white additive Gaussian having zero mean and variance $\sigma_{n}^{2}=1$. Therefore, $R_{n}=\sigma_{n}^{2} I$. The only a priori infor-
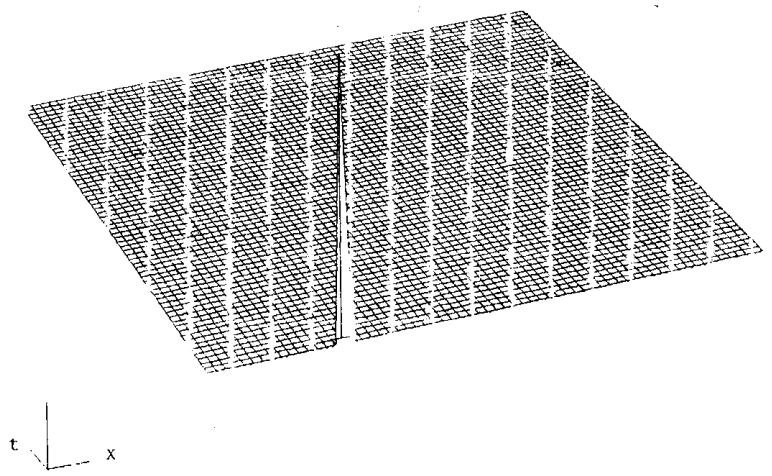

Fig. 4. Ideal diffractor point.

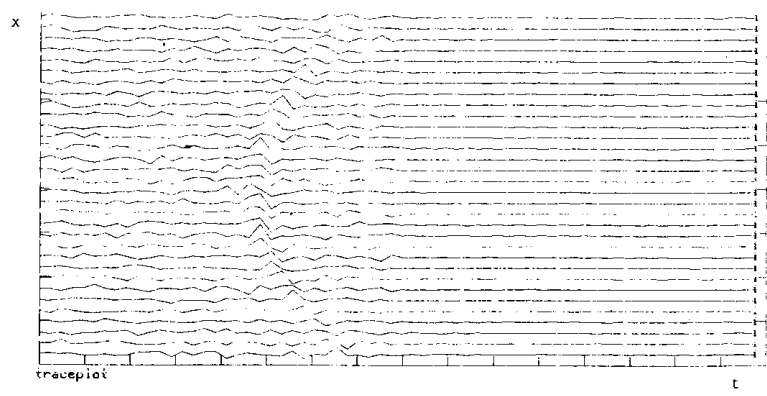

Fig. 5. Seismic traces corrupted by white Gaussian noise.

mation available about $\boldsymbol{x}$ is that it is Gaussian distributed and white $\left(R_{x}=\sigma_{x}^{2} I\right)$, having variance $\sigma_{x}^{2}=1$. The $a$ priori mean $\overline{\boldsymbol{x}}$ is given by $\bar{x}_{i}=1$ for the impulse position and $\bar{x}_{i}=0$ otherwise. Such a choice of $\bar{x}$ is reasonable because an impulse is expected. Note that the value of $\bar{x}_{i}$ at the impulse position is 1 , whereas the real impulse strength is 5 . The real wave propagation velocity is 3200 $\mathrm{m} / \mathrm{s}$. However, it is assumed to be unknown. The only $a$ priori information available about the velocity is its mean $\bar{c}=3000 \mathrm{~m} / \mathrm{s}$ and its variance $\sigma_{c}^{2}=500$. The initial guess about the velocity is $2000 \mathrm{~m} / \mathrm{s}$ which is far from the real velocity.

The conventional technique is to apply a matched downward extrapolator operator to our surface data. The operator has the form [1], [10]

$$
W^{\prime}\left(k_{x}, k\right)= \begin{cases}\exp \left(i z_{0} \sqrt{k^{2}-k_{x}^{2}}\right) & k^{2} \geq k_{x}^{2} \\ \exp \left(-z_{0} \sqrt{k^{2}-k_{x}^{2}}\right) & k^{2}<k_{x}^{2} .\end{cases}
$$

The results of the inverse extrapolation are shown in Fig. 6. They are far away from the expected ones, mainly due to the wrong velocity used and due to the existence of noise. If we know the actual velocity, the results are much better, as is shown in Fig. 7. The Wiener filter has the following form [9], [10] in the wavenumber-frequency domain:

$$
H_{w}\left(k_{x}, k\right)=\frac{H^{*}\left(k_{x}, k\right)}{\left|H\left(k_{x}, k\right)\right|^{2}+\left|\frac{S_{n n}\left(k_{x}, k\right)}{S_{x x}\left(k_{x}, k\right)}\right|^{2}}
$$




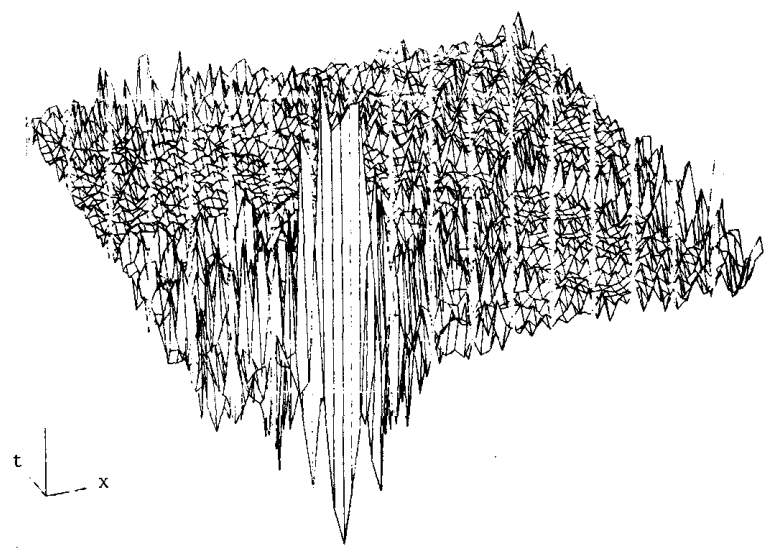

Fig. 6. Backward extrapolation of the signal of Fig. 5 with wrong velocity $2000 \mathrm{~m} / \mathrm{s}$ (instead of $3200 \mathrm{~m} / \mathrm{s}$ ).

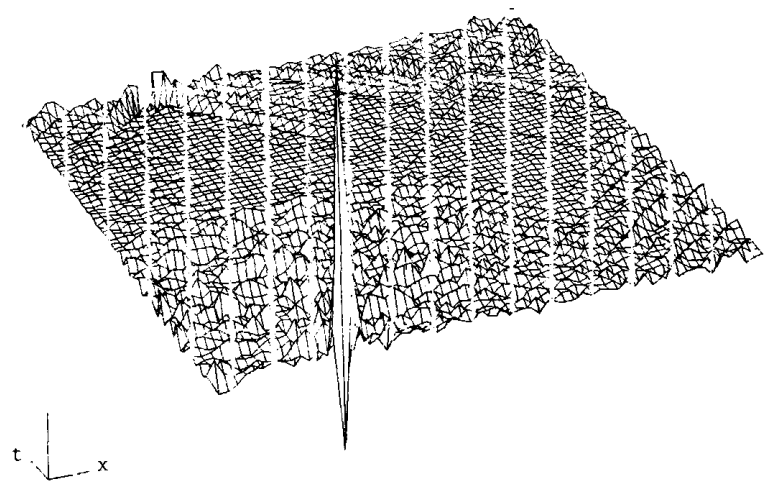

Fig. 7. Backward extrapolation of the signal of Fig. 5 with correct velocity $3200 \mathrm{~m} / \mathrm{s}$.

where $S_{n n}, S_{x x}$ are the power spectra of $x, n$, respectively. In the case of white noise $n$ and on the assumption that $x$ is white noise too, the Wiener filter degenerates to the matched downward extrapolator:

$$
H_{w}\left(k_{x}, k\right)=\frac{W^{\prime}\left(k_{x}, k\right)}{1+\left|\frac{S_{n n}\left(k_{x}, k\right)}{S_{x x}\left(k_{x}, k\right)}\right|^{2}}=\frac{1}{1+a} W\left(k_{x}, k\right) .
$$

Thus, the application of the Wiener filter has no meaning in this case. The MAP estimation gives much better results than those of the inverse filter. A slightly modified operator $H\left(k_{x}, k\right)$ has been used in the MAP estimation:

$$
H^{\prime}\left(k_{x}, k\right)=\left\{\begin{array}{c}
\exp \left(-i z_{0} \sqrt{k^{2}-k_{x}^{2}}+i \omega \frac{z_{0}}{c}\right) \\
k^{2} \geq k_{x}^{2} \\
\exp \left(-z_{0} \sqrt{k^{2}-k_{x}^{2}}+i \omega \frac{z_{0}}{c}\right) \\
k^{2}<k_{x}^{2} .
\end{array}\right.
$$

The term $e^{i \omega(z 0 / c)}$ advances the pressures measured on the surface by $-\left(z_{0} / c\right)$ in time:

$$
P^{\prime}(x, z=0, t)=P\left(x, z=0, t-\frac{z_{0}}{c}\right) .
$$

The advanced pressures are used many times in geophysics [1]. Their use improves the convergence properties of the MAP estimation with respect to the velocity since it advances all hyperbolas of different velocities to the time $t=0$. Thus, difference in velocity $c$ affects only the curvature of the hyperbolas, as is shown in Fig. 9. The technique employed in MAP estimation was to estimate iteratively the velocity $c$ by (31) and then to estimate iteratively $x$ by (32). The whole algorithm has been implemented in the wavenumber-frequency domain. The convolutions of the form $H x,(\partial H / \partial c) x, H^{T} x$ have been implemented by using a two-dimensional Fourier transform [12]. The exact form of $\partial H / \partial c$ used has the following form in the wavenumber-frequency domain:

$$
\frac{\partial H\left(k_{x}, k\right)}{\partial c}=\left\{\begin{array}{c}
\frac{i \omega^{2} z_{0}}{c^{3} \sqrt{k^{2}-k_{x}^{2}}} \exp \left(i z_{0} \sqrt{k^{2}-k_{x}^{2}}\right) \\
k^{2} \geq k_{x}^{2} \\
-\frac{\omega^{2} z_{0}}{c^{3} \sqrt{k_{x}^{2}-k^{2}}} \exp \left(-z_{0} \sqrt{k_{x}^{2}-k^{2}}\right) \\
k^{2}<k_{x}^{2}
\end{array}\right.
$$

and for the advanced pressure operator,

$$
\begin{aligned}
& \left(\left(\frac{i z_{0} \omega^{2}}{c^{3} \sqrt{k^{2}-k_{x}^{2}}}-\frac{i \omega z_{0}}{c^{2}}\right)\right.
\end{aligned}
$$

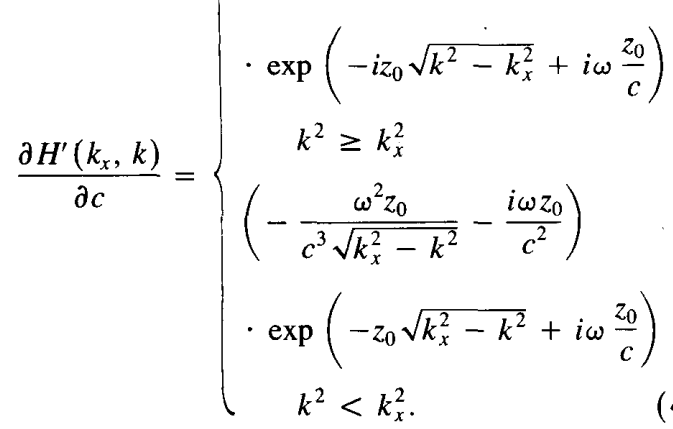

Proofs of (42), (43) can be found in the Appendix. The initial value about the velocity is $2000 \mathrm{~m} / \mathrm{s}$. The initial value about $\boldsymbol{x}$ is its a priori mean $\overline{\boldsymbol{x}}$. The convergence of the algorithm is quite fast. In ten iterations, the velocity estimate has been found to be $3215 \mathrm{~m} / \mathrm{s}$ which is very close to the actual one $(3200 \mathrm{~m} / \mathrm{s})$. The estimate of $x$ was calculated in another ten iterations and is shown in Fig. 8. It is much better than the result of Fig. 6. It is also better than the result of Fig. 7, although there is not a big difference between them. This is explained by the fact that MAP estimation of $\boldsymbol{x}$ (for correct velocity) is a 


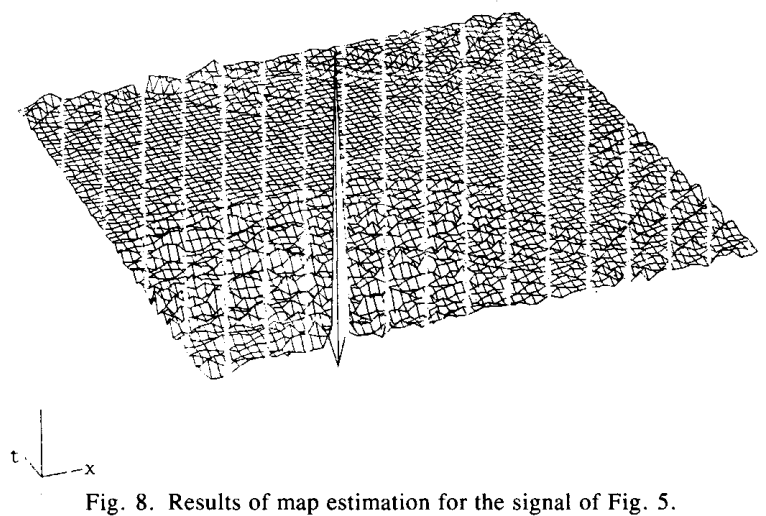

modified Wiener filter with the a priori mean $\bar{x}$ included as in (30). In this example, the Wiener filter degenerates to the inverse filter and the only improvement of Fig. 8 over Fig. 6 is due to the a priori mean $\bar{x}$.

The second case to be considered is that of the colored noise of Fig. 10. The velocity of the surface wave is 4000 $\mathrm{m} / \mathrm{s}$ and the velocity of the diffracted wave is $3000 \mathrm{~m} / \mathrm{s}$. This is not realistic since surface waves usually travel with less velocity than the diffracted waves. The velocity filtering is based on this assumption [2]. We have chosen such a surface wave velocity because in this case, the spectra of signal and noise overlap and colored noise cannot be removed by velocity filtering. The same $a$ priori information about the wavefield $\boldsymbol{x}$ and the velocity $c$ has been used, as in the case of the white noise. The autocorrelation matrix $R_{n}$ of the colored noise has been obtained by taking the inverse Fourier transform of the power spectrum of the noise.

The result of inverse extrapolation with velocity 2000 $\mathrm{m} / \mathrm{s}$ is very bad, as shown in Fig. 11. This is due to the colored noise and to the wrong migration velocity used. The results of the extrapolation with correct velocity (if known) are shown in Fig. 12. There is a significant improvement over Fig. 11, but the effects of the colored noise are still very large. They can be reduced, though, by Wiener filtering with correct velocity (if known) as is shown in Fig. 13. The MAP estimation has been used as in the case of the white noise. An estimate of the velocity $3177 \mathrm{~m} / \mathrm{s}$ has been found in ten iterations. An estimate of $\boldsymbol{x}$, found in ten iterations, is shown in Fig. 14. There is a clear improvement over the inverse extrapolation and the Wiener filtering. The MAP estimation greatly depends on the choice of the a priori mean $\bar{x}$ and of the covariance matrices, $R_{x}, R_{n}$, as is obvious from (28), (29). The matrices $R_{x}, R_{n}$ weight our confidence on the data $y$ or on the a priori information $\bar{x}$, as can be seen from (28), (29). Especially in the case of white noise, the term $R_{x} H^{T} R_{n}^{-1}$ becomes $\left(\sigma_{x}^{2} / \sigma_{n}^{2}\right) H^{T}$ and the ratio $\sigma_{x}^{2} / \sigma_{n}^{2}$ controls our confidence on the data $\boldsymbol{y}$ or on the a priori information $\overline{\boldsymbol{x}}$. In the same case, the ratio $\sigma_{c}^{2} / \sigma_{n}^{2}$ controls our confidence on the a priori velocity information $\bar{c}$ or on the data $y$, as can be seen in (29).

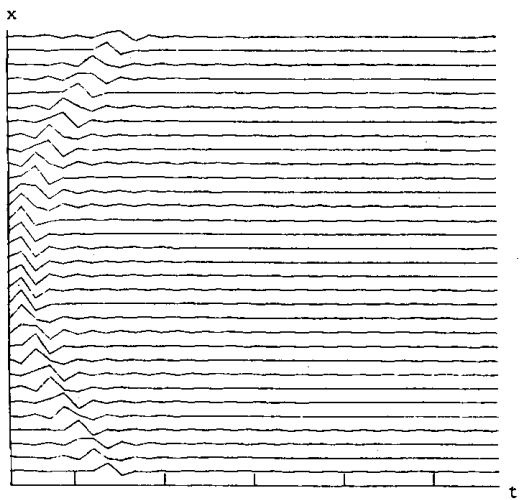

(a)

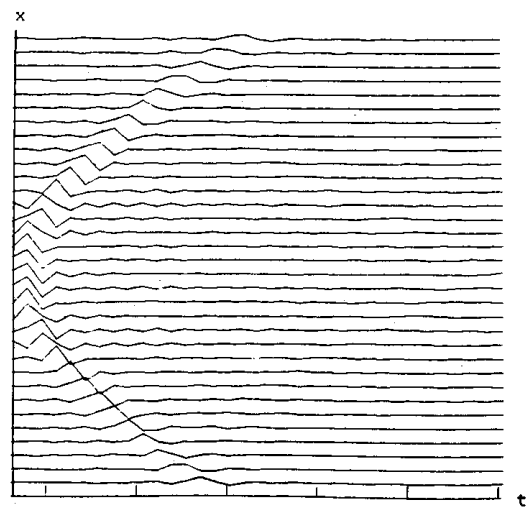

(b)

Fig. 9. Time-advanced recorded data. (a) Velocity $4000 \mathrm{~m} / \mathrm{s}$. (b) Velocity $2000 \mathrm{~m} / \mathrm{s}$.

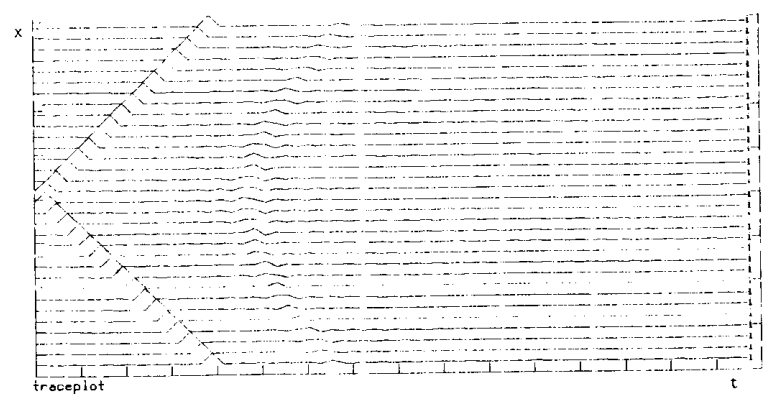

Fig. 10. Seismic traces corrupted by surface wave having velocity 4000 $\mathrm{m} / \mathrm{s}$ and amplitude double the diffracted signal.

The iterative algorithms used are computationally intensive, mainly due to the calculation of 2-D convolution by means of 2-D fast Fourier transforms. The size of the data used in our example was $64 \times 64$. $0.5 \mathrm{~min}$ CPU time per iteration is needed on a VAX 780 machine, using the Fortran 77 language and UNIX operating system. We have not used any other optimization techniques because the steepest descent iteration converged fairly quickly 


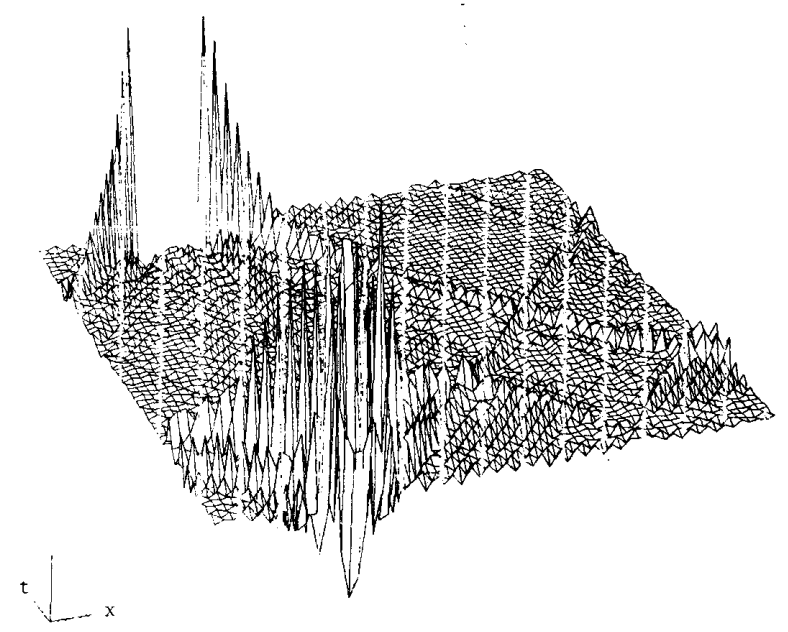

Fig. 11. Backward extrapolation of the signal of Fig. 10 with wrong velocity $2000 \mathrm{~m} / \mathrm{s}$ (instead of $3200 \mathrm{~m} / \mathrm{s}$ ).

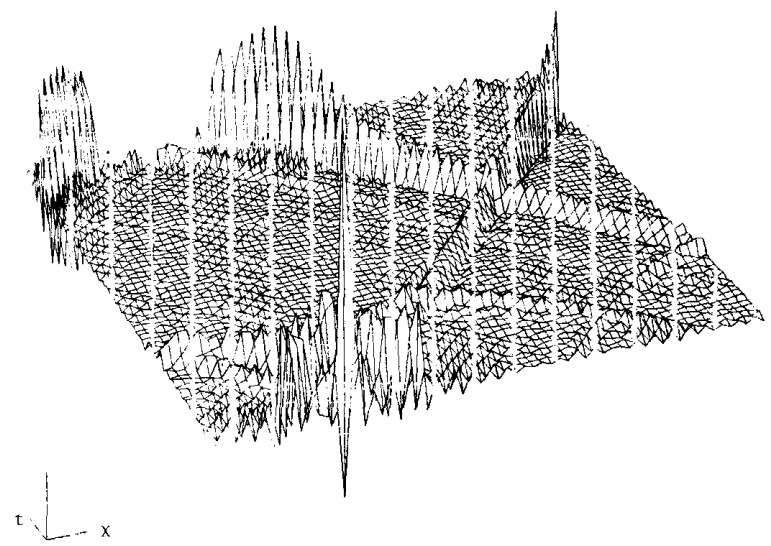

Fig. 12. Backward extrapolation of the signal of Fig. 10 with correct velocity $3200 \mathrm{~m} / \mathrm{s}$.

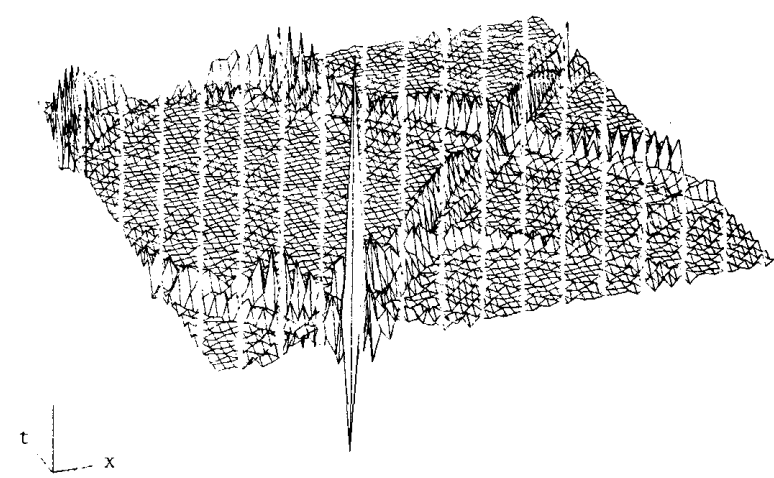

Fig. 13. Results of the Wiener filter of the signal of Fig. 10.

(usually in less than ten iterations) and because our main intention was to demonstrate the correctness of our approach. We believe, though, that other schemes will reduce the number of iterations, but not the computational

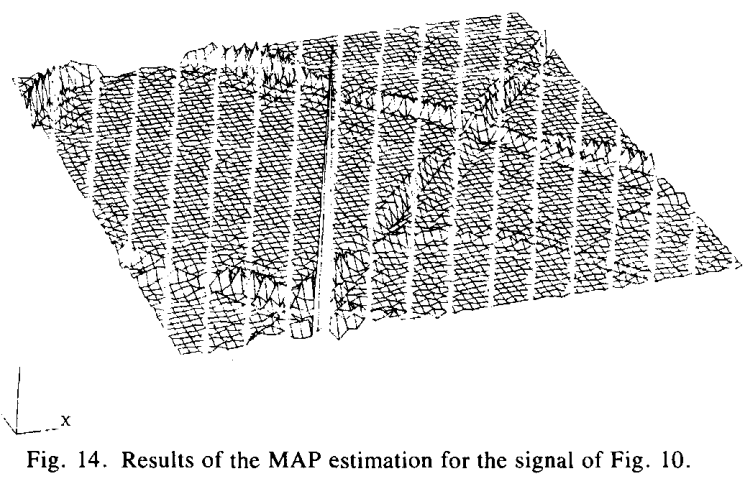

load per iteration (in fact, it will be slightly increased). Thus, we do not expect great computational savings from other optimization techniques.

\section{Conclusions}

Bayesian estimation techniques have been proposed for use in the wave inversion problems. These techniques are proven to be superior to known methods like the direct inversion or Wiener filtering. Their superiority comes from the fact that they exploit all the a priori information available and that they also provide means to estimate characteristics of the media, like the velocity of wave propagation. Most of them are iterative algorithms. This gives a flexibility in the techniques, but it also causes problems when the convergence rate of the algorithm is slow. Generally speaking, the algorithms are quite computationally intensive since they use two-dimensional fast Fourier transforms for their implementation. Sophisticated techniques can be used to speed up algorithm convergence. We have not used them since our primary aim was to show that the algorithms work and that they are superior to the known ones. A further limitation of the theory and the example presented here is that they treat only the one-layer case. The example used, although very simple, proves the validity of our approach. The Bayesian estimation in wavefield inversion has already been extended for the multilayered media model, which is much more realistic. The theory and simulation results are presented in [20].

\section{APPENDIX}

Derivatives of the Extrapolation Operator in Two Dimensions

The forward wave extrapolation operator in the two dimensions has the following form in the space-time domain [2]:

$$
\begin{aligned}
h\left(x, z_{0}, t\right) & =\frac{\partial}{\partial z}\left(\frac{\delta\left(t-\frac{r}{c}\right)}{-2 \pi r}\right) \\
r & =\sqrt{x^{2}+z_{0}^{2}}
\end{aligned}
$$

$\delta$ : delta function 
PITAS AND VENETSANOPOULOS: BAYESIAN ESTIMATION OF MEDIUM PROPERTIES

795

and the following form in the wavenumber-frequency domain:

$$
H\left(k_{x}, k\right)= \begin{cases}\exp \left(-i z_{0} \sqrt{k^{2}-k_{x}^{2}}\right) & k^{2} \geq k_{x}^{2} \\ \exp \left(-z_{0} \sqrt{k_{x}^{2}-k^{2}}\right) & k^{2}<k_{x}^{2} .\end{cases}
$$

It is well known that the Fourier transform pair is

$$
h\left(x, z_{0}, t\right)=\int_{-\infty}^{\infty} \int_{-\infty}^{\infty} H\left(k_{x}, k\right) e^{i k_{x} x} e^{i \omega t} d k_{x} d \omega .
$$

Substituting (A2) in (A3) gives

$$
\begin{aligned}
h\left(x, z_{0}, t\right)= & \int_{-\infty}^{\infty} \int_{-\infty}^{-\omega / c} \exp \left(-z_{0} \sqrt{k_{x}^{2}-\frac{\omega^{2}}{c^{2}}}\right) \\
& \cdot e^{-i k_{x} x} e^{i \omega t} d k_{x} d \omega \\
& +\int_{-\infty}^{\infty} \int_{-\omega / c}^{\omega / c} \exp \left(-i z_{0} \sqrt{\frac{\omega^{2}}{2}-k_{x}^{2}}\right) \\
& \cdot e^{i k_{x} x} e^{i \omega t} d k_{x} d \omega \\
& +\int_{-\infty}^{\infty} \int_{\omega / c}^{\infty} \exp \left(-z_{0} \sqrt{k_{x}^{2}-\frac{\omega^{2}}{c^{2}}}\right) \\
& \cdot e^{i k_{x} x} e^{i \omega t} d k_{x} d \omega .
\end{aligned}
$$

The derivative of $h\left(x, z_{0}, t\right)$ with respect to $c$ is

$$
\frac{\partial h}{\partial c}=\frac{\partial}{\partial c} \int_{-\infty}^{\infty} \int_{-\infty}^{\infty} H\left(k_{x}, z_{0}, k\right) e^{i k_{x} x} e^{i \omega t} d k_{x} d \omega .
$$

By combining (A4) and (A5), we find

$$
\begin{aligned}
\frac{\partial h}{\partial c}= & \int_{-\infty}^{\infty} \frac{\partial}{\partial c}\left[\int_{-\infty}^{-\omega / c} \exp \left(-z_{0} \sqrt{k_{x}^{2}-\frac{\omega^{2}}{c^{2}}}\right)\right. \\
& \left.\cdot e^{i k_{x} x} d k_{x}\right] e^{-i \omega t} d \omega \\
& +\int_{-\infty}^{\infty} \frac{\partial}{\partial c}\left[\int_{-\omega / c}^{\omega / c} \exp \left(-i z_{0} \sqrt{\frac{\omega^{2}}{c^{2}}-k_{x}^{2}}\right)\right. \\
& \left.\cdot e^{i k_{x} x} d k_{x}\right] e^{-i \omega t} d \omega \\
& +\int_{-\infty}^{\infty} \frac{\partial}{\partial c}\left[\int_{\omega / c}^{\infty} \exp \left(-z_{0} \sqrt{k_{x}^{2}-\frac{\omega^{2}}{c^{2}}}\right)\right. \\
& \left.\cdot e^{i k_{x} x} d k_{x}\right] e^{i \omega t} d \omega \\
= & \int_{-\infty}^{\infty}\left[\int_{-\infty}^{-\omega / c} \frac{z_{0} \omega^{2}}{c^{3} \sqrt{k_{x}^{2}-\frac{\omega^{2}}{c^{2}}}}\right.
\end{aligned}
$$$$
\cdot \exp \left(-z_{0} \sqrt{k_{x}^{2}-\frac{\omega^{2}}{c^{2}}}\right) e^{i k_{x} x} d k_{x}
$$$$
\left.+e^{-i x(\omega / c)} \frac{\omega}{c^{2}}\right] e^{i \omega t} d \omega
$$$$
+\int_{-\infty}^{\infty}\left[\int_{-\omega / c}^{\omega / c} \frac{i z_{0} \omega^{2}}{c^{3} \sqrt{\frac{\omega^{2}}{c^{2}}-k_{x}^{2}}}\right.
$$$$
\exp \left(-i z_{0} \sqrt{k_{x}^{2}-\frac{\omega^{2}}{c^{2}}}\right) e^{i k_{x} x} d k_{x}
$$$$
\left.-e^{i x(\omega / c)} \frac{\omega}{c^{2}}-e^{-i x(\omega / c)} \frac{\omega}{c^{2}}\right] e^{i \omega t} d \omega
$$$$
+\int_{-\infty}^{\infty}\left[\int_{\omega / c}^{\infty}-\frac{z_{0} \omega^{2}}{c^{3} \sqrt{k_{x}^{2}-\frac{\omega^{2}}{c^{2}}}}\right.
$$$$
\cdot \exp \left(-z_{0} \sqrt{k_{x}^{2}-\frac{\omega^{2}}{c^{2}}}\right) e^{i k_{x} x} d k_{x}
$$$$
\left.+e^{i x(\omega / c)} \frac{\omega}{c^{2}}\right] e^{i \omega t} d \omega
$$

$$
\begin{aligned}
& \int_{-\infty}^{\infty} \int_{-\infty}^{-\omega / c}-\frac{z_{0} \omega^{2}}{c^{3} \sqrt{k_{x}^{2}-\frac{\omega^{2}}{c^{2}}}} \\
& +\exp \left(-z_{0} \sqrt{\left.k_{x}^{2}-\frac{\omega^{2}}{c^{2}}\right) e^{i k_{x} x}} e^{i \omega t} d k_{x} d \omega\right. \\
& +\int_{-\infty}^{\infty} \int_{-\omega / c}^{\omega / c} \frac{i z_{0} \omega^{2}}{c^{3} \sqrt{\frac{\omega^{2}}{c^{2}}-k_{x}^{2}}} \\
& +\exp \left(-i z_{0} \sqrt{\left.k_{x}^{2}-\frac{\omega^{2}}{c^{2}}\right) e^{i k_{x} x}} e^{i \omega t} d k_{x} d \omega\right. \\
& +\int_{-\infty}^{\infty} \int_{\omega / c}^{\omega / c}-\frac{z_{0} \omega^{2}}{c^{3} \sqrt{k_{x}^{2}}-\frac{\omega^{2}}{c^{2}}} \\
& \cdot \exp \left(-z_{0} \sqrt{\left.k_{x}^{2}-\frac{\omega^{2}}{c^{2}}\right) e^{i k_{x} x}} e^{i \omega t} d k_{x} d \omega .\right.
\end{aligned}
$$

(Aw) 
Thus, the Fourier transform of $\partial h / \partial c$ is

$$
F\left(\frac{\partial h}{\partial c}\right)=\left\{\begin{array}{l}
\frac{i \omega^{2} z_{0}}{c^{3} \sqrt{\frac{\omega^{2}}{c^{2}}-k_{x}^{2}}} \exp \left(-i z_{0} \sqrt{\frac{\omega^{2}}{c^{2}}-k_{x}^{2}}\right) \\
-\frac{k^{2} \geq k_{x}^{2}}{c^{3} \sqrt{\frac{\omega^{2}}{c^{2}}-k_{x}^{2}}} \exp \left(-z_{0} \sqrt{k_{x}^{2}-\frac{\omega^{2}}{c^{2}}}\right) \\
k^{2}<k_{x}^{2} .
\end{array}\right.
$$

When time advanced pressures are used, then a different extrapolator is used:

$$
H^{\prime}\left(k_{x}, z_{0}, k\right)=H\left(k_{x}, z_{0}, k\right) e^{i \omega z 0 / c}
$$

for an advance by $-\left(z_{0} / c\right) \mathrm{ms}$ in time.

In this case, the Fourier transform of $\partial h^{\prime} / \partial c$ is

$$
F\left(\frac{\partial h^{\prime}}{\partial c}\right)=\left\{\begin{array}{l}
\left(\frac{i z_{0} \omega^{2}}{c^{3} \sqrt{\frac{\omega^{2}}{c^{2}}-k_{x}^{2}}-\frac{i \omega z_{0}}{c^{2}}}\right) \\
\exp \left(-i z_{0} \sqrt{k^{2}-k_{x}^{2}}\right) \exp \left(i \omega z_{0} / c\right) \\
k^{2} \geq k_{x}^{2} \\
\left(-\frac{z_{0} \omega^{2}}{c^{3} \sqrt{k_{x}^{2}-\frac{\omega^{2}}{c^{2}}}-\frac{i \omega z_{0}}{c^{2}}}\right) \\
\exp \left(-z_{0} \sqrt{k_{x}^{2}-k^{2}}\right) \exp \left(i \omega z_{0} / c\right) \\
k^{2}<k_{x}^{2} .
\end{array}\right.
$$

\section{REFERENCES}

[1] A. J. Berkhout, Seismic Migration. Amsterdam, The Netherlands: Elsevier, 1982.

[2] E. A. Robinson and M. T. Silvia, Digital Foundations of Time Series Analysis: Vol. 2-Wave Equation Space-Time Processing. San Francisco, CA: Holden-Day, 1984
[3] E. A. Robinson and S. Treitel, Geophysical Signal Analysis. Englewood Cliffs, NJ: Prentice-Hall, 1980.

[4] W. A. Schneider, "Integral formulation for migration in two and three dimensions," Geophys., vol. 43, pp. 49-76, Feb. 1978.

[5] D. E. Robinson and M. Wing, "Lateral deconvolution of ultrasonic beams," Ultrason. Imaging, vol. 6, pp. 1-12, 1984.

[6] A. Papoulis, Systems and Transforms with Applications in Optics. New York: McGraw-Hill, 1968.

[7] R. L. Sengbush and M. R. Foster, "Optimum multichannel velocity filters," Geophys., vol. 33, pp. 11-35, Feb. 1968.

[8] D. De Vries and A. J. Berkhout, "Velocity analysis based on minimum entropy," Geophys., vol. 49, pp. 2132-2142, Dec. 1984.

[9] A. Papoulis, Probability, Random Variables and Stochastic Processes. New York: McGraw-Hill, 1965.

[10] A. J. Berkhout and D. W. Van Wulfften Palthe, "Migration in the presence of noise," Geophys. Prospecting, vol. 28, pp. 372-383, 1980.

[11] M. Fatemi and A. C. Kak, "Ultrasonic B-scan imaging: Theory of image formation and techniques for restoration," Ultrason. Imaging, vol. 2 , Jan. 1980.

[12] H. Van Trees, Detection, Estimation and Modulation Theory, Vol. 1. New York: Wiley, 1968.

[13] A. Tarantola and B. Valette, "Inverse problems = quest for information,"'J. Geophys., pp. 159-170, 1982.

[14] W. Menke, Geophysical Data Analysis: Discrete Inverse Theory. New York: Academic, 1984.

[15] J. Mendel, Optimal Seismic Deconvolution: An Estimation Based Approach. New York: Academic, 1983.

[16] B. J. Uscinski, The Elements of Wave Propagation in Random Media. New York: McGraw-Hill, 1977.

[17] B. R. Hunt, "Bayesian methods in nonlinear digital image restoration," IEEE Trans. Comput., vol. C-26, pp. 219-229, Mar. 1977.

[18] J. Kowalik and M. R. Osborne, Methods for Unconstrained Optimization Problems. Amsterdam, The Netherlands: Elsevier, 1968.

[19] R. Fox, Optimization Methods for Engineering Design. Reading. MA: Addison-Wesley, 1971

[20] I. Pitas and A. N. Venetsanopoulos "Bayesian estimation in seismic migration," IEEE Trans. Acoust., Speech, Signal Processing, in press.

Ioannis Pitas (S'80-M'85), for a photograph and biography, see p. 264 of the February 1988 issue of this Transactions.

Anastasios N. Venetsanopoulos (S'66-M'69-SM'79), for a photograph and biography, see p. 264 of the February 1988 issue of this TRANSACTIONS. 